\title{
SENSING SINGLE CELL CONTRACTILITY UTILIZING MOIRE FRINGES
}

\author{
$X$. Zheng $^{1}$, H. Surks ${ }^{2}$, and X. Zhang ${ }^{*}$ \\ ${ }^{1}$ Boston University, Boston, Massachusetts, USA \\ ${ }^{2}$ Tufts Medical Center, Boston, Massachusetts, USA
}

\begin{abstract}
Abnormal vascular cell contractile performance is a hallmark of cardiovascular diseases. Conventional cell force measurement technique requires individually tracking the sensing units and complex computation efforts for further studying cell contractility. We developed instead a robust and simple compact optical moiré system that measures phase changes encoded in carrier moiré patterns generated from two layers of gratings. Cell mechanics study including cell contractile forces, stress and strain distributions during normal and abnormal cell contractions can thus be conveniently analyzed. The distinct signals from moiré patterns in longitudinal and transverse directions revealed abnormal cell mechanical contractility linked to cardiovascular disease.
\end{abstract}

\section{INTRODUCTION}

The mechanical interaction of cells with their extracellular matrix (ECM) is fundamentally important for cell division, growth, phagocytosis, apoptosis and migration [1], [2]. Bidirectional chemical and mechanical signals between cells and their ECM result in the regulation of cellular processes and the remodeling of the extracellular environment. The mechanical interaction, presented as cell traction forces, is therefore a crucial factor in physiological processes such as development and wound healing. For example, the physiological regulation of vascular cell contraction is critical to the maintenance of normal vascular function. The regulation of cell function occurs as a result of the tension in the cytoskeleton and the distortion of the cells. Abnormal contraction forces distribution in vascular cells is an early indication of vascular diseases including hypertension, atherosclerosis and myocardial infarction. Any study of single vascular cells function requires a way to quantify mechanical forces in a precise, dynamic and reproducible manner with high fidelity and accuracy.

Many of the previous approaches for cell force mapping are not satisfactory since they require fixation, dehydration and induce undesirable external stimulation to the cells. As a result such approaches cannot make the cell force determination capable of revealing the contractile state of cells. Recently, micro and nano patterned polymeric substrates have been introduced for measuring cell traction forces [3]. These new methods introduce a local determination of the traction forces upon discretelized adhesion areas between the cells and their underlying polymeric substrates. In these techniques the discrete displacement vectors are derived by tracking and monitoring deflections of each individual microstructure. Despite this, these methods require intensive computation and expertise to locate individual sensing units, track and derive their displacement fields. Moreover, with fixed numerical aperture of the objective lens, the deflection or motion of individual sensing unit is often in the range of sub-micrometers. As a result tracking such deflection or motion of each individual high-density micro structure is time-consuming and thus cannot meet the demands for real-time monitoring of cellular and subcellular behaviors.

We have previously demonstrated a method for cell traction force measurement based on optical moiré effect [4]. Moiré methods have been used extensively in engineering as diagnostic tools to measure microscopic strain and stress because of their high sensitivity to spatial displacements and distortions within small structures. They have also been used in conventional lithography and soft lithography fabrications to determine the alignment state between periodic substrates. Inspired by this phenomena and its ability to measure in-plane displacement and distortion, we have reported that the magnification effects of 1-dimentional (1D) and 2-dimentional (2D) moiré patterns are capable of magnifying cells contraction forces. The employed interferometric moiré offers precise and high resolution moiré mapping. This technique takes the advantage of moiré magnification effect by calculating the moiré fringe distortions and map into the distortions of polymeric periodic substrate. The distortion of moiré fringes provides a magnified map of cell force distributions in whole field. The magnification factor of the optical moiré typically ranges from 2$20 \times$ by adjusting the relative angle between two coherent beams. The periodicity of the moiré pattern is determined by the intersecting angle of the two beams and the orientation of the periodic substrate. However, the reported system was limited for practical use due to the requirement for intereference facilities. Optical alignment added difficulty for achieving on-chip compact moiré system for real time observation of cell contractility. In addition, the method used for retrieving force distribution was based on geometric fringe centering approach which prevents further automation of the system into laboratory use.

\section{THEORY}

Interferometric moiré offers precise and high resolution moiré mapping. We have demonstrated that by illuminating the periodic substrate with two symmetric light beams [Figure. 1(a)], the moiré pattern can be generated by two symmetrical beams diffracted by periodic substrate. The periodicity of the moiré pattern is determined by intersecting angle of the two beams and the orientation of the periodic substrate, i.e.,

$$
\frac{1}{d_{m}} \exp (-j \varphi)=\frac{2 \sin \alpha}{\lambda} \exp (-j \psi)-\frac{1}{d}
$$

Incident angle $\alpha$ and $\psi$ which is the orientation of the polymeric periodic substrate, are two adjustable parameters that determine the orientation and spatial periodicity of the moiré patterns. The symmetrical arrangement of the two incident beams ensured that the moiré pattern is only sensitive to in-plane deformation on gratings whereas out-of-plane distortions carried by two beams are canceled out by being diffracted through the substrate. If replacing one plane wave with a thin periodic substrate with the same period, by adjusting the relative orientation of the two substrates, moiré patterns can be generated by the diffraction of the coherent wave through two separated layers of the periodic substrates. Description of such compact moiré pattern generation system is elaborated as follows.

The two grating patterns $T_{1}(x), T_{2}(x)$ (with grating lines perpendicular to the x-axis), with spatial period $d_{1}$ and $d_{2}$ can be expressed in Fourier series [5]:

$$
T_{1}(x)=\sum_{n=-\infty}^{+\infty} T_{n}\left(\text { in } \frac{2 \pi}{d_{1}}\left(x \cos \theta_{1}+y \sin \theta_{1}\right)\right)
$$




$$
T_{2}(x)=\sum_{n=-\infty}^{+\infty} T_{n}\left(\text { in } \frac{2 \pi}{d_{2}}\left(x \cos \theta_{2}+y \sin \theta_{2}\right)\right)
$$

$\theta_{1} \theta_{2}$ are the absolute orientation of the two substrates with respect to the $\mathrm{X}$ axis and their relative orientation is denoted in the insert of Figure [1(b) and (c)], as $\theta$, namely: $\theta=\theta_{1}-\theta_{2}$. For simplicity, let the two periodic substrates have the same spatial periodicity and their relative orientation is $\theta$. This assumes collimated illumination, i.e., a plane incoming wavefront. From far-field Fresnel Diffraction theory, the two grating patterns intercept [Figure 1(b)] to have a diffraction field which can be expressed as:

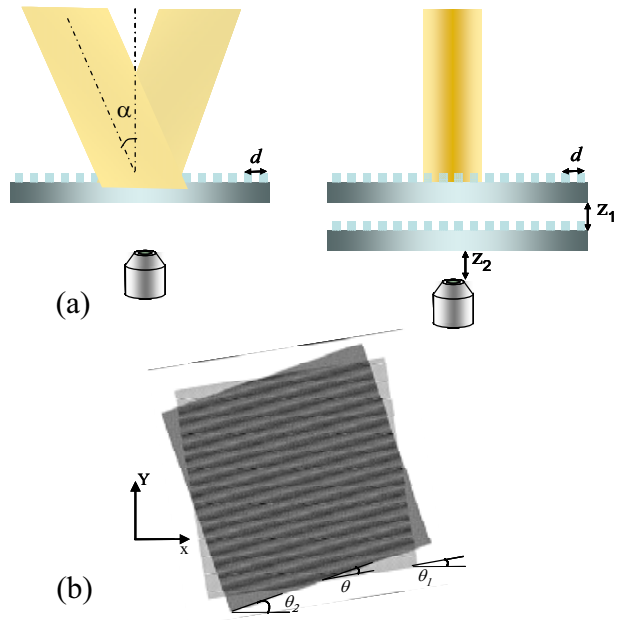

Figure 1: Representative unwrapped phase map in cardiac myocyte (a) and converted wrapped 3-D phase map in cardiac myosyte (b). The phase is linearly correlated with the force.

Where $z_{1}$ is the distance between the two substrates, $z_{2}$ is the distance between the second substrate to the observation plane, shown in Figure. 1(b). $n$ and $m$ are integer numbers. Part of the expression which has the lowest frequencies is the one that describes the moiré pattern. When the two periodic substrates have a small misalignment angle $\theta\left(\theta<30^{\circ}\right)$, the spatial distribution of the moiré pattern in space can be described as [5]

$$
\begin{aligned}
& U^{\prime}\left(x_{3}, y_{3}\right) \approx \sum_{n=-\infty}^{\infty} T\left(\frac{n}{d}\right) T\left(-\frac{n}{d}\right) \\
& =\exp \left\{-i \pi \frac{n^{2} \lambda}{d^{2}}\left(z_{1}+4 z_{2} \sin ^{2} \theta\right)\right\} \exp \left\{i 2 \pi n / \frac{y_{3} d}{2 \sin \theta}\right\}
\end{aligned}
$$

The above equation shows that the moiré fringes can be treated as a grating pattern with spatial period $d / 2 \sin \theta$, with contrast changing along the $z$ axis with an interval of $d_{T}=2(d / \sin \theta)^{2} / \lambda$. At distances that are integer multiples of $d_{T}$, the blur owing to the grating's diffraction is minimized, meaning the contrast of the fringe pattern attains a local maximum. By adjusting the gap between the two gratings to integer multiples of $d_{T}$, we can avoid diffraction by the gratings and justify our approach.

The periodicity of the moire pattern is invariant with the distance between the two periodic substrates, i.e.,

$$
d_{m}=d / 2 \sin \left(\frac{\theta}{2}\right)
$$

With small angle approximation, the last equation is simplified as $d_{m}=d / \theta$. Eq. (5) is the same as Eq. (1), except that the reference layer in interferometric configuration is the intereference pattern. The orientation and spatial period of the moiré pattern corresponding to the relative angle $\theta$ between two periodic substrates of equal spatial period is numerically plotted in Figure 2. In Figure 2 the periodicity of moiré pattern versus the angle $\theta$ is shown for five grating periods ( $3 \mu \mathrm{m}, 4 \mu \mathrm{m}, 5 \mu \mathrm{m}, 6 \mu \mathrm{m}, 7 \mu \mathrm{m}$ from bottom to top respectively). The orientation of the moiré fringes ranges from $80^{\circ}-90^{\circ}$ as the relative orientation of the periodic substrates increases from $0-30^{\circ}$. It can be observed that the spatial period of moiré pattern decreases when the angle $\theta$ increases. However, this decreasing is not linear, but three distinct regions can be observed. The first region is between 0 to 10 degrees: the magnification factor is relatively large and they diminish quickly as $\psi$ increases. This is, therefore, a very sensitive region. In the second region, with $\theta$ ranging from 10 to 20 degrees, the variations in $d_{m}$ are moderate (smaller than a dozen $\mu \mathrm{m})$. For example, when the period of the arrays is $d=4 \mu \mathrm{m}$, the period of the moiré fringes goes from $40 \mu \mathrm{m}$ (at $\theta=5^{\circ}$ ) to $11 \mu \mathrm{m}$ (at $\theta=20^{\circ}$ ). In the third region, from 20 to 30 degrees, the variations of $d_{m}$ are small, approaching to a constant asymptotic value, noted as the least sensitive region.

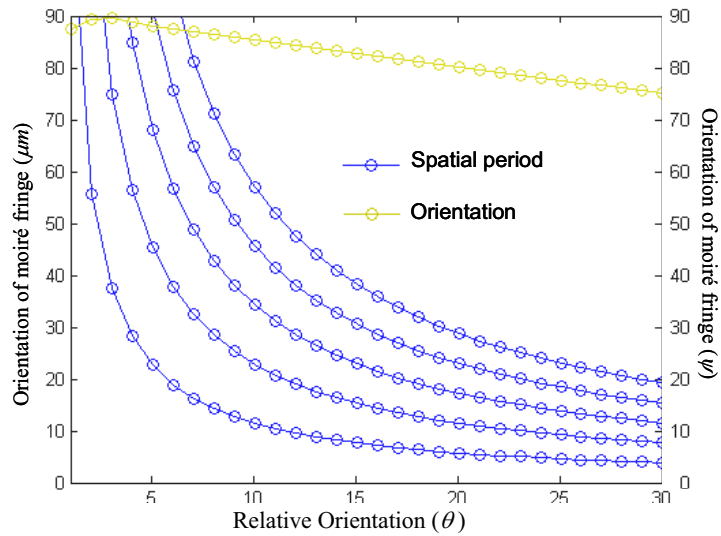

Figure 2: Magnification factor and orientation of moiré fringes versus the relative angle of two superimposing gratings of periods. The red curve represents orientation of the moiré pattern; the blue curve represents magnification factor.

\section{EXPERIMENT AND RESULTS}

The experimental setup shown in Figure 3 for our compact 2D moiré mapping system includes an illumination source of $1.5-\mathrm{mW}$ 633-nm He-Ne laser (Research Electro-Optics Inc, a beam expander, a CCD video camera (Pixelink 623), an inverted microscope (Nikon), a digital computer with MATLAB programs for automated moiré fringes analysis, and two identical PDMS periodic substrate substrates mounted on the translational stage. The grating lines and grids patterns are separated by sidewalls. The reference layer can be shifted by a translational stage so that 1D and 2D moiré patterns can be conveniently switched. The sidewalls also serve as guidance to separate periodic pillar arrays so that the cardiac mycoytes will be aligned in the direction of the wall. The cell culturing substrate was sealed into a polycarbonate flow perfusion chamber with an internal volume of $30 \mathrm{~mL}$. Cell culture media with Rat aortic smooth muscle cells were injected through the inlet of the perfusion chamber with the culturing substrate. The plane of the reference substrate is kept parallel to that of the cell culture substrate and the orientation is controlled by a rotation stage. Testing marks on the sidewalls were designed to help locate the periodic features and relative orientation between the two substrates. The glass environmental chamber is maintained at $37.2^{\circ} \mathrm{C}$ with $5 \% \mathrm{CO}_{2}$ concentration. 
The cell culture chip and reference layer were fabricated by following a pressure-assisted replica molding process. AZ 9260 (AZ Electronics, Inc) was spin-coated on glass slides $(2 \mathrm{~cm} \times 2 \mathrm{~cm})$ at $3000 \mathrm{rpm}$ for $60 \mathrm{~s}$ and soft-baked for $2.5 \mathrm{~min}$ at $100{ }^{\circ} \mathrm{C}$. After being exposed under a UV light at $1550 \mathrm{~mJ}$, the samples were developed in AZ 400K diluted as 1:4 (AZ Electronics) for about 20 s. PDMS prepolymer (Sylgard 184, Dow Corning, 10:1 base solution to curing agent) was then spin-coated on patterned photoresist on glass slide at $1000 \mathrm{rpm}$ for $5 \mathrm{~s}$, put into vacuum oven for $10 \mathrm{~min}$ for bubble removal and soft baked at $65^{\circ} \mathrm{C}$ for $2 \mathrm{~h}$.

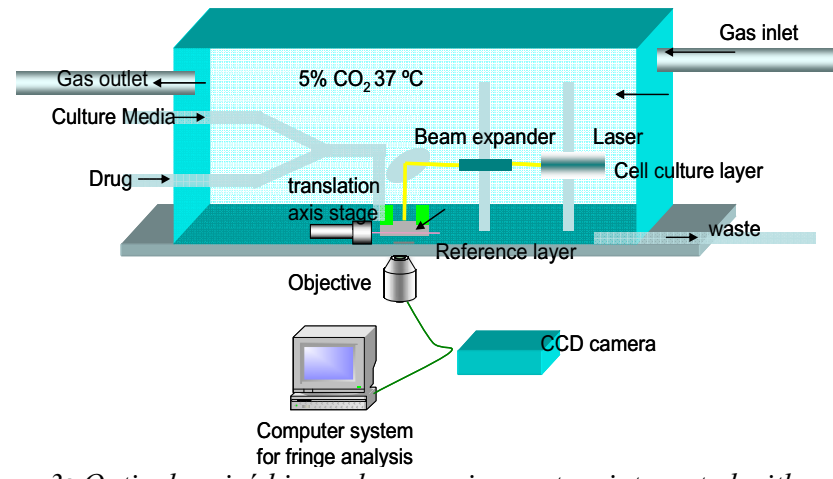

Figure 3: Optical moiré biomechno sensing system integrated with cell culture facilities.

The resulting thickness of the PDMS was approximately $200 \mu \mathrm{m}$ upon removal from the glass substrate. And the resulting diameter of the PDMS posts is $2 \mu \mathrm{m}$ with height of $4 \mu \mathrm{m}$.

The cell culture chip needs to be activated by extracellular matrix (ECM) protein (Laminin for cardiac myocyte and fibronectin for smooth muscle cell) to render cell adhesion to the substrate. This process was performed by inverted micro contact printing ECM proteins on top of the cell culture substrate [6]. After releasing the PDMS from the mold, the cell culture layer was treated in oxygen plasma in a Plasma Asher (TePla M4L PVA Plasma Asher) at 150w for 2 mins. This oxygen plasma treatment made the PDMS surface change from being hydrophobic to being hydrophilic which facilitates the absorption of fibronectin in the PBS buffer solution. The PDMS stamps were cut from thick layered PDMS chunk, immersed in $50 \mu \mathrm{g} / \mathrm{mL}$ fibronectin PBS buffer solution, and then washed and blow dried under a nitrogen gun. These protein coated PDMS stamps were finally put in conformal contact with the surface oxidized cell culture samples for $5 \mathrm{~min}$ and then carefully deconstructed. For cardiac myocytes culture, the polymer substrate was sterilized and $15 \mathrm{mmol} \mathrm{l}^{-1}$ of laminin.

To demonstrate the operation of our moiré contouring system based on Fourier carrier fringe analysis, we propose to map the anisotropy of cardiac myocyte contraction driven by electric current. Rat cardiac myocytes were isolated from Wistar rat hearts according to a previously described protocol [7]. The laminin solution was removed and the cells were plated on the PDMS substrate for $2 \mathrm{~h}$ to allow for cell attachment. After that, the polymeric substrate was gently rinsed with fresh culture media to remove the unattached cells. The cells were incubated in $5 \% \mathrm{CO}_{2}$ at $37^{\circ} \mathrm{C}$, and the culture media was changed every $72 \mathrm{~h}$. Cardiac myocytes contraction induced distortion on testing grids patterns consisting of short cantilever like pillar arrays, and thus the measurement of contractility of single cardiac myocytes was performed by plating isolated living cells on our chip with shortcantilever like grids pattern confined within sidewalls. The cells formed focal adhesions on the tops of the deflectable grids patterns consisting of the posts and were thus free to contract unhindered by their attachments causing movement of the posts. The perfusion chamber was perfused with $1.2 \mathrm{mM} \mathrm{Ca}^{2+}$ Tyrode buffer solution and maintained at $37{ }^{\circ} \mathrm{C}$ for $24 \mathrm{~h}$ on the substrate. The cells were electrically stimulated to contract at a frequency of $5 \mathrm{~Hz}$ from IonOptix. During the experiment, the orientation of interference patterns was adjusted to contain a small angle $\left(8^{\circ}\right)$ with respect to one $(\mathrm{X})$ direction of the spatial periodicity of the substrate. The grating line was translated to be directly on top of the cell culturing layer to serve as reference grating. Figure 4(a) shows a typical 1D initial undistorted moiré pattern. Figsure 4 (b) and (c) show typical moiré pattern at the peak of cardiac myocyte contraction in two perpendicular directions respectively.

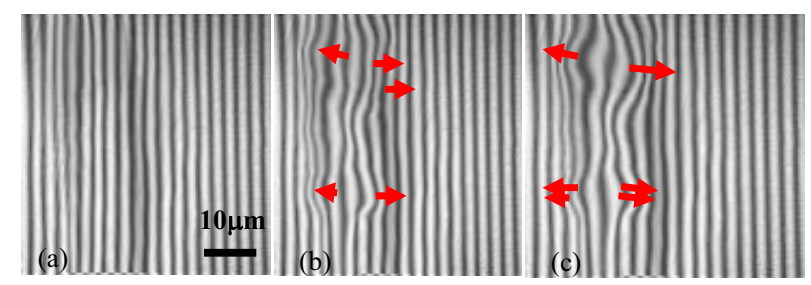

Figure 4: Moiré fringe evolution in cardiac myosytes contraction. (a) Initial fringe pattern. (b) 0.5 period of cell contraction. (c) 1 period of cell contraction.
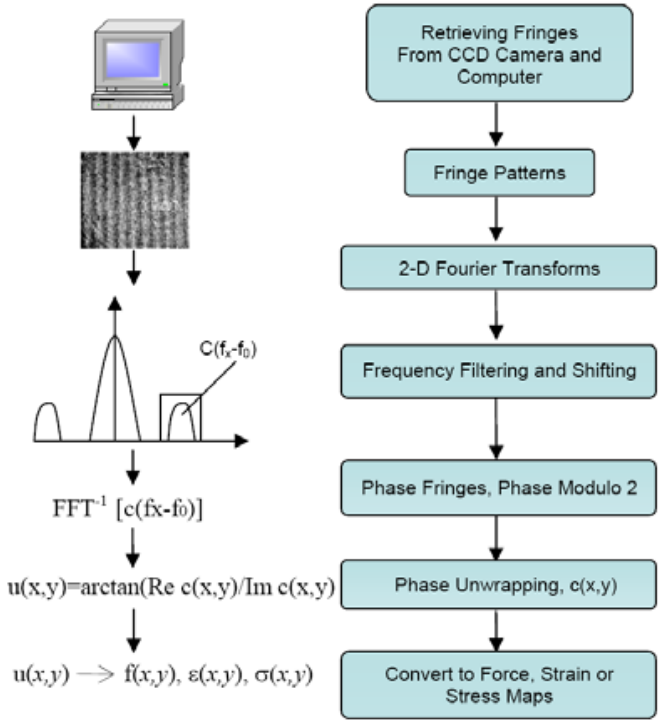

Figure 5: Scheme for automated fringe analysis procedure based on carrier fringe Fourier transform method for cell force mapping.

With the stimulation at $5 \mathrm{~Hz}$ at 2.5 volts, the contraction period of cardiac myocytes is $0.2 \mathrm{~s}$. The distortion of $1 \mathrm{D}$ moiré pattern clearly reveals distortion induced by cardiac myocyte contraction. To compute the contraction force distribution from the moiré maps, Fourier transform was applied to the image (Figure 5). A reverse transform on the masked data after frequency filtering then carries noise-free phase information. Each pixel contains a complex number of which the phase can be calculated. The phase records the position of a pixel under the distortion pattern, since a $\pi$ change in phase is equivalent to shifting from a null to a peak in the distortion fringes. Phase unwrapping was then carried out by subtracting any $2 \pi$ phase jumps. Figure 6 (a) shows the unwrapped phase change pattern from the moiré pattern when 
cell contraction was at its peak upon stimulation. Unwrapped phase patterns directly revealed cell contraction distribution in the whole cell. Utilizing the load-displacement relationship characterized from nanoindentation, of which the Young's Modulus was determined to be $3 \mathrm{MPa}$, the traction force distribution was converted from the phase distortion map [Figure. 6(b)]. 1D moiré pattern in two directions can be achieved by rotating the grating pattern by $90^{\circ}$ clockwise.

We studied the moiré pattern evolution during smooth muscle cell spreading and cell contraction in response to agonist [8]. The amount of cell contraction in response to agonist is closely related to level of cell traction forces already established in cells. Therefore, it is important to study the cell contractile forces evolution prior to stimulating the cells with contractile agonists. We examined the cell force evolution through moiré pattern evolution and investigated the contractile forces in response to agonist.

(a)
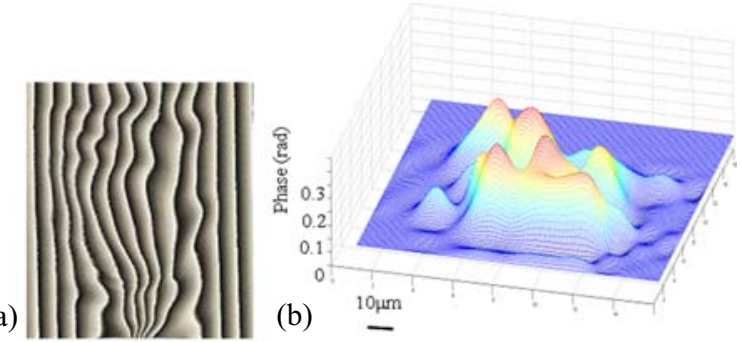

Figure 6: Representative unwrapped phase map in cardiac myocyte (a) and converted wrapped 3-D phase map in cardiac myosyte (b). The phase is linearly correlated with the force. (a)

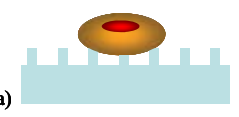

Baseline contraction

(b)

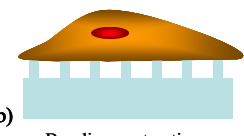

(c)

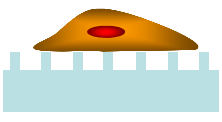

Rest in serum free media

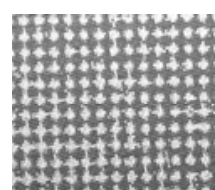

$0 \mathrm{hr}$

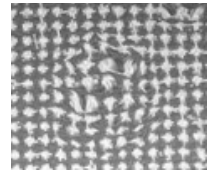

$24 \mathrm{hr}$

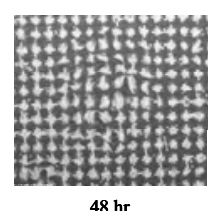

$48 \mathrm{hr}$
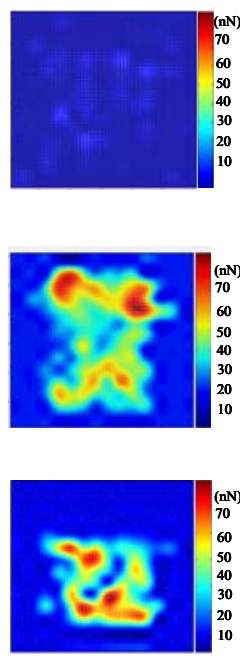

Figure 7: Cell patterning and moiré pattern evolution: (a) VSMC cell just seeded on the substrate. (b) Cell spread out and traction forces were developed. (c) Cell relaxed in serum free media.

The force exerted by cell traction can then be conveniently studied through moiré pattern evolution. The substrate was slightly rotated with respect to the first substrate to form an angular moiré pattern and the resulting spatial period of moiré pattern was found to be to be $8 \mu \mathrm{m}$. The reference layer consisted of periodic pillar patterns with aspect ratio of 2 . After being plated on the cell culture layer, the cells started to establish focal adhesions and further spread out on the fibronectin coated periodic patterns.

The corresponding moiré image reveals undistorted moiré grids patterns, as shown in Figure 7 (a)-(c). As the cells started to spread out, the moiré patterns started to deform for approximately $12 \mathrm{~h}$ until its fully distorted pattern. The evolution of moiré fringes confirmed that the smooth muscle cells fully spread out and that the traction force accumulates due to the increase in cytoskeleton tension dependent on serum. To map cell traction forces during cell spreading, a 2D Fourier transform was applied and the phase change was retrieved from the carrier fringes with a spatial period of $8 \mu \mathrm{m}$. The moiré image magnified and recorded the original deformation map into deformed carrier fringe patterns. The distortion of the fringes was then converted to a phase distortion map $u(x, y)$, followed by being converted into a force map $f(x, y)$.

\section{CONCLUSIONS}

We have demonstrated a real time optical moiré mapping system using coherent light. The premise of this work is that the optical moiré interface will provide automatic whole-field mapping of cell mechanical interactions with its living environment in real time. Optical moiré techniques have unique properties such as its automatic displacement and strain contouring and its magnification effect for small strains and displacements. Given these unique properties, this new approach would be an improvement over existing techniques since it can be integrated with the existing engineered cell culture substrates and provide a direct contour mapping of the displacements and strain distribution without tracking individual sensing units.

\section{ACKNOWLEDGEMENTS}

We would like to acknowledge National Science Foundation (CBET 0933653, CMMI 0826191, and CMMI 0239163) for the support of this research.

\section{REFERENCES}

[1]. K. A. Beningo and Y. L. Wang "Flexible substrata for the detection of cellular traction forces", Trends in Cell Biology, 12, 79 (2002).

[2] N. Q. Balaban, U. S. Schwarz, D. Riveline, P. Goichberg, G. Tzur, I. Sabanay, D. Mahalu, S. Safran, A. Bershadsky, L. Addadi, and B. Geiger, "Force and focal adhesion assembly: a close relationship studied using elastic micropatterned substrates," Nature Cell Biology, 3, 466 (2001).

[3] J.L. Tan, et al.,"Cells lying on a bed of microneedles: An approach to isolate mechanical force", Proceedings of the National Academy of Sciences of the United States of America, 100, 1484 (2003).

[4] X. Zheng and X. Zhang, "An optical Moiré technique for cell traction force mapping", Journal of Micromechanics and Microengineering, 18, 125006 (2008).

[5] O. K. a. I. Glatt, The Physics of Moiré Metrology. New York: John Wiley and Sons, 1990.

[6] C. D. James, R. C. Davis, L. Kam, H. G. Craighead, M. Isaacson, J. N. Turner, and W. Shain, "Patterned protein layers on solid substrates by thin stamp microcontact printing," Langmuir, 14 (1998).

[7] J. Tytgat, "How to Isolate Cardiac Myocytes," Cardiovascular Research, vol. 28, pp. 280-283, Feb 1994.

[8] A. P. Somlyo and A. V. Somlyo, "Signal-transduction and regulation in smooth-muscle," Nature, 372, 231 (1994).

\section{CONTACT}

*Xin Zhang; 617-358-2702, xinz@bu.edu 